PROCEEDINGS OF THE

AMERICAN MATHEMATICAL SOCIETY

Volume 133, Number 6, Pages 1573-1580

S 0002-9939(04)07704-4

Article electronically published on December 6, 2004

\title{
SURFACES, SUBMANIFOLDS, AND ALIGNED FOX REIMBEDDING IN NON-HAKEN 3-MANIFOLDS
}

\author{
MARTIN SCHARLEMANN AND ABIGAIL THOMPSON
}

(Communicated by Ronald A. Fintushel)

\begin{abstract}
Understanding non-Haken 3-manifolds is central to many current endeavors in 3-manifold topology. We describe some results for closed orientable surfaces in non-Haken manifolds, and extend Fox's theorem for submanifolds of the 3 -sphere to submanifolds of general non-Haken manifolds. In the case where the submanifold has connected boundary, we show also that the $\partial$-connected sum decomposition of the submanifold can be aligned with such a structure on the submanifold's complement.
\end{abstract}

\section{INTRODUCTION}

A closed orientable irreducible 3-manifold $N$ is called Haken if it contains a closed orientable incompressible surface; otherwise $N$ is non-Haken. In Section 2 we describe some results for surfaces in non-Haken manifolds. Generalizing a theorem of Fox ([F]), we show in Section 3 that a 3-dimensional submanifold of a nonHaken manifold $N$ is homeomorphic either to a handlebody complement in $N$ or the complement of a handlebody in $S^{3}$. Sections 2 and 3 are independent, but both represent progress towards understanding submanifolds of non-Haken manifolds. In Section 4 we combine the techniques from Section 2 with the results from Section 3 to show that if the submanifold $M \subset N$ is $\partial$-reducible and has connected boundary, then the embedding can be chosen to align a full collection of separating $\partial$-reducing disks in $M$ with similar disks in the complement of $M$.

\section{HANDLEBODIES IN NON-HAKEN MANIFOLDS}

Let $N$ be a closed orientable 3-manifold, $F$ a closed orientable surface of nontrivial genus imbedded in $N$. Recall that $F$ is compressible if there exists an essential simple closed curve on $F$ that bounds an imbedded disk $D$ in $N$ with interior disjoint from $F . D$ is a compressing disk for $F$.

Definition 1. Suppose $F$ is a separating closed surface in an orientable irreducible closed 3-manifold N.F is reducible if there exists an essential simple closed curve on $F$ that bounds compressing disks on both sides of $F$. The union of the two compressing disks is a reducing sphere for $F$.

Received by the editors September 28, 2003 and, in revised form, February 10, 2004. 2000 Mathematics Subject Classification. Primary 11Y16, 57M50; Secondary 57M25.

This research was supported in part by NSF grants.

(C)2004 American Mathematical Society Reverts to public domain 28 years from publication 
Suppose $\mathbf{S}$ is a collection of disjoint reducing spheres for $F$. A reducing sphere $S \in \mathbf{S}$ is redundant if a component of $F-\mathbf{S}$ that is adjacent to $S \cap F$ is planar. $\mathbf{S}$ is complete if, for any disjoint reducing sphere $S^{\prime}, S^{\prime}$ is redundant in $\mathbf{S} \cup S^{\prime}$.

Let $\sigma(\mathbf{S})$ denote the number of components of $F-\mathbf{S}$ that are not planar surfaces.

Since $N$ is irreducible, any sphere in $N$ is necessarily separating. Suppose a reducing sphere $S^{\prime}$ is added to a collection $\mathbf{S}$ of disjoint reducing spheres. If $S^{\prime}$ is redundant, the number of non-planar complementary components in $F$ is unchanged, since $S^{\prime}$ necessarily separates the component of $F-\mathbf{S}$ that it intersects and the union of two planar surfaces along a single boundary component is still planar. If $S^{\prime}$ is not redundant, then the number of non-planar complementary components in $F$ increases by one. Thus we have:

Lemma 2. Suppose $\mathbf{S} \subset \mathbf{S}^{\prime}$ are two collections of disjoint reducing spheres for $F$ in $N$. Then $\sigma(\mathbf{S}) \leq \sigma\left(\mathbf{S}^{\prime}\right)$. Equality holds if and only if each sphere $S^{\prime}$ in $\mathbf{S}^{\prime}-\mathbf{S}$ is redundant in $S^{\prime} \cup \mathbf{S}$. In particular, $\mathbf{S}$ is complete if and only if for every collection $\mathbf{S}^{\prime}$ such that $\mathbf{S} \subset \mathbf{S}^{\prime}, \sigma(\mathbf{S})=\sigma\left(\mathbf{S}^{\prime}\right)$.

Let $H$ be a handlebody imbedded in $N . H$ has an unknotted core if there exists a pair of transverse simple closed curves $c, d \subset \partial H$ such that $c \cap d$ is a single point, $d$ bounds an imbedded disk in $H$ and $c$ (the core) bounds an imbedded disk in $N$ transverse to $\partial H$. Note that the interior of the latter imbedded disk may intersect $H$.

Lemma 3. Let $F$ be a connected, closed, separating, orientable surface in a closed orientable irreducible 3-manifold $N$. Suppose that $F$ has compressing disks to both sides. Then at least one of the following must hold:

(1) $F$ is a Heegaard surface for $N$.

(2) $N$ is Haken.

(3) There exist disjoint compressing disks for $F$ on opposite sides of $F$.

Proof. The proof is an application of the generalized Heegaard decomposition described in $[\mathrm{ST}]$. Since $F$ is compressible to both sides, we can construct a handle decomposition of $N$ starting at $F$ so that $F$ appears as a "thick" surface in the decomposition. If $F$ is not a Heegaard surface, then this decomposition contains a "thin" surface $G$ adjacent to $F$. If $G$ is incompressible in $N$, then $N$ is Haken. If $G$ is compressible we apply [CG] to obtain the required disjoint compressing disks for $F$.

Theorem 4. Let $H$ be a handlebody of genus $g$ imbedded in a closed orientable irreducible non-Haken 3-manifold $N$. Let $G$ be the complement of $H$ in $N$. Let $F=\partial H=\partial G$. Suppose $F$ is compressible in $G$. Then at least one of the following must hold:

(1) The Heegaard genus of $N$ is less than or equal to $g$.

(2) $F$ is reducible.

(3) $H$ has an unknotted core.

Proof. The proof is by induction on the genus of $H$. If $g=1$, then the result of compressing $F$ into $G$ is a 2 -sphere, necessarily bounding a ball in $N$. If a ball it bounds lies in $G$, then the Heegaard genus of $N$ is $\leq 1$. If a ball it bounds contains $H$, then $H$ is an unknotted solid torus in $N$, and so it has an unknotted core. 
Suppose then that $\operatorname{genus}(H)=g>1$ and assume inductively that the theorem is true for handlebodies of genus $g-1$. Suppose that $G$, the complement of $H$, has compressible boundary. If $G$ is a handlebody, then $G \cup_{F} H$ is a Heegaard splitting of genus $g$ and we are done. So suppose $G$ is not a handlebody. Then by Lemma 3 there are disjoint compressing disks on opposite sides of $F$, say $D$ in $H$ and $E$ in $G$. Without loss of generality, we can assume that $D$ is non-separating. Compress $H$ along $D$ to obtain a new handlebody $H_{1}$ with boundary $F_{1}$; let $G_{1}$ be the complement of $H_{1}$.

If $\partial E$ is inessential in $F_{1}$, then it bounds a disk in $H_{1} \subset H$ as well, so $F$ is reducible.

If $\partial E$ is essential in $F_{1}$, then $E$ is a compressing disk in $G_{1}$ and so we can apply the inductive hypothesis to $H_{1}$. If 1 or 3 holds, then it holds for $H$, and we are done. Suppose instead $F_{1}$ is reducible. Let $\mathbf{S}$ be a collection of disjoint reducing spheres for $F_{1}$ chosen to maximize $\sigma$ among all possible such collections and then, subject to that condition, further choose $\mathbf{S}$ to minimize $|E \cap \mathbf{S}|$. Clearly $E \cap \mathbf{S}$ contains no closed curves, else replacing a subdisk lying in the disk collection $\mathbf{S} \cap G_{1}$ with an innermost disk of $E-\mathbf{S}$ would reduce $|E \cap \mathbf{S}|$. Similarly, we have

Claim 1. Suppose $\epsilon$ is an arc component of $\partial E-\mathbf{S}$ and $F_{0}$ is the component of $F_{1}-\mathbf{S}$ in which $\epsilon$ lies. If $\epsilon$ separates $F_{0}$ (so the ends of $\epsilon$ necessarily lie on the same component of $\partial F_{0}$ ), then neither component of $F_{0}-\epsilon$ is planar.

Proof of Claim 1. Let $c_{0}$ be the closed curve component of $\partial F_{0} \subset \mathbf{S} \cap F_{1}$ on which the ends of $\epsilon$ lie and, of the two arcs into which the ends of $\epsilon$ divide $c_{0}$, let $\alpha$ be adjacent to a planar component of $F_{0}-\epsilon$. Then the curve $\epsilon \cup \alpha$ clearly bounds a disk in both $G_{1}$ and $H_{1}$, and then so does the curve $c^{\prime}=\epsilon \cup\left(c_{0}-\alpha\right)$. Let $S^{\prime}$ be a sphere in $N$ intersecting $F_{1}$ in $c^{\prime}$ and $S_{0}$ be the reducing sphere in $\mathbf{S}$ containing $c_{0}$. Replacing $S_{0}$ with $S^{\prime}$ (or just deleting $S_{0}$ if $c^{\prime}$ is inessential in $F_{1}$ ) gives a new collection $\mathbf{S}^{\prime}$ of disjoint reducing spheres, intersecting $\partial E$ in at least two fewer points. Moreover $\sigma\left(\mathbf{S}^{\prime}\right)=\sigma(\mathbf{S})$ since the only change in the complementary components in $F_{1}$ is to add to one component and delete from another a planar surface along an arc in the boundary. Then the collection $\mathbf{S}^{\prime}$ contradicts our initial choice for $\mathbf{S}$, a contradiction that proves the claim.

Let $H^{\prime}$ be the closed complement of $\mathbf{S}$ in $H_{1}$, so $H^{\prime}$ is itself a collection of handlebodies.

Claim 2. Either $F$ is reducible or $\partial H^{\prime}$ is compressible in $N-H^{\prime}$.

Proof of Claim 2. If $\partial E$ is disjoint from $\mathbf{S}$ and is inessential in $\partial H^{\prime}$, then $\partial E$ bounds a disk in $H^{\prime}$, hence in $H$, so $F$ is reducible. If $\partial E$ is disjoint from $\mathbf{S}$ and is essential in $\partial H^{\prime}$, then $E$ compresses $\partial H^{\prime}$ in $N-H^{\prime}$, verifying the claim. Finally, if $E$ intersects $\mathbf{S}$, consider an outermost disk $A$ cut off from $E$ by $\mathbf{S}$. According to Claim 1, this disk, together with a subdisk of $\mathbf{S}$, constitute a disk $E^{\prime}$ that compresses $\partial H^{\prime}$ in $N-H^{\prime}$, proving the claim.

Following Claim 2, either $F$ is reducible or the inductive hypothesis applies to a component $H_{0}$ of $H^{\prime}$. If 2 holds for $H_{0}$, then consider a reducing sphere $S$ for $H_{0}$, isotoped so that the curve $c=S \cap \partial H_{0}$ is disjoint from the disks $\mathbf{S} \cap H_{0}$. The disk $S-H_{0}$ may intersect $H_{1}$; by general position with respect to the dual 1-handles, each component of intersection is a disk parallel to a component of $\mathbf{S} \cap H_{1}$. But each such disk can be replaced by the corresponding disk in $\mathbf{S}-H_{1}$ so that in the 
end $c$ also bounds a disk in $N-H_{1}$. After this change, $S$ is a reducing sphere for $F_{1}$ in $N$ and, since $c$ is essential in $H_{0}, \sigma(\mathbf{S} \cup S)>\sigma(\mathbf{S})$, contradicting our initial choice for $\mathbf{S}$. Thus in fact 1 or 3 holds for $H_{0}$, hence also for $H$.

In the specific case $N=S^{3}$, we apply precisely the same argument, combined with Waldhausen's theorem [W] on Heegaard splittings of $S^{3}$, to obtain:

Corollary 5. Let $H$ be a handlebody imbedded in $S^{3}$, and suppose $G$, the complement of $H$, has compressible boundary. Then either $H$ has an unknotted core or the boundary of $H$ is reducible.

This corollary is similar to ([MT], Theorem 1.1), but no reimbedding of $S^{3}-H$ is required.

\section{COMPlEMENTS OF HANDLEBODIES IN NON-HAKEN MANIFOLDS}

In $[\mathrm{F}$ (see also $[\mathrm{MT}]$ for a brief version) Fox showed that any compact connected 3-dimensional submanifold $M$ of $S^{3}$ is homeomorphic to the complement of a union of handlebodies in $S^{3}$. We generalize this result to non-Haken manifolds, showing that a submanifold $M$ of a non-Haken manifold $N$ has an almost equally simple description, that is, $M$ is homeomorphic to the complement of handlebodies either in $S^{3}$ or in $N$.

Definition 6. Let $N$ be a compact irreducible 3-manifold, and let $M$ be a compact 3 -submanifold of $N$. We will say the complement of $M$ in $N$ is standard if it is homeomorphic to a collection of handlebodies or to $N \#$ (collection of handlebodies). (We regard $B^{3}$ as a handlebody of genus 0.)

Note that in the latter case $M$ is actually homeomorphic to the complement of a collection of handlebodies in $S^{3}$.

Theorem 7. Let $N$ be a closed orientable irreducible non-Haken 3-manifold, and let $M$ be a connected compact 3-submanifold of $N$ with non-empty boundary. Then $M$ is homeomorphic to a submanifold of $N$ whose complement is standard.

Proof. The proof will be by induction on $n+g$ where $n$ is the number of components of $\partial M$ and $g$ is the genus of $\partial M$, that is, the sum of the genera of its components. If $n+g=1$, then $\partial M$ is a single sphere. Since $N$ is irreducible, the sphere bounds a 3-ball in $N$. So either $M$ or its complement is a 3-ball and in either case the proof is immediate.

To verify the inductive step, suppose first that $\partial M$ has multiple components $T_{1}, \ldots, T_{n}, n \geq 2$. Each component $T_{i}$ must bound a distinct component $J_{i}$ of $N-M$ since each must be separating in the non-Haken manifold $N$. Let $M^{\prime}=M \cup J_{n}$; by inductive assumption $M^{\prime}$ can be reimbedded so that its complement is stan-

dard. After the reimbedding, remove $J_{n}$ from $M^{\prime}$ to recover a homeomorph of $M$ and adjoin $J_{1}$ (now homeomorphic either to a handlebody or to $N \#$ (handlebody)) instead. Reimbed the resulting manifold so that its complement is standard and remove $J_{1}$ to recover $M$, now with standard complement.

Henceforth we can therefore assume that $\partial M$ is connected and not a sphere. Since $N$ is non-Haken there exists a compressing disk $D$ for $\partial M$ in $N-\partial M$; the compressing disk lies either in $M$ or in its closed complement $J$. 
Case 1. $\partial D$ is non-separating on $\partial M$.

If $D$ lies inside $M$, compress $M$ along $D$ to obtain $M^{\prime}$ and use the induction hypothesis to find an imbedding of $M^{\prime}$ with standard complement. Reconstruct $M$ by attaching a trivial 1-handle to $M^{\prime}$, thus simutaneously attaching a trivial 1-handle to the complement.

If $D$ lies outside $M$, attach a 2-handle to $M$ corresponding to $D$ to obtain $M^{\prime}$, whose connected boundary has lower genus. Invoking the inductive hypothesis, imbed $M^{\prime}$ in $N$ with standard complement. Reconstruct $M$ from $M^{\prime}$ by removing a co-core of the attached 2-handle, thus adding a 1-handle to the complement of $M^{\prime}$.

Case 2. $\partial D$ is separating on $\partial M$.

Suppose $D$ lies outside $M$. Then $D$ also separates the closed complement $J$ of $M$ into two components, $J_{1}$ and $J_{2}$, since $H_{2}(N)=0$. Denote the components of $\partial M-\partial D$ by $\partial_{1} \subset J_{1}$ and $\partial_{2} \subset J_{2}$, both of positive genus. Let $M^{\prime}=M \cup J_{2}$. Reimbed $M^{\prime}$ so that its complement is standard. The boundary of $M^{\prime}$ consists of $\partial_{1}$ together with a disk. Since the complement of $M^{\prime}$ is standard, there is a nonseparating compressing disk $D^{\prime}$ for $\partial M^{\prime}$ contained in the complement of $M^{\prime} . D^{\prime}$ is also a non-separating compressing disk for the reimbedded $\partial M$ (which is contained in $\left.M^{\prime}\right)$. Apply case 1 to this new imbedding of $M$.

We can now suppose that the only compressing disks for $\partial M$ are separating compressing disks lying inside $M$. Choose a family $\mathbf{D}$ of such $\partial$-reducing disks for $M$ that is maximal in the sense that no component of $M^{\prime}=M-\mathbf{D}$ is itself $\partial$-compressible. Since each compressing disk is separating, genus $\left(\partial M^{\prime}\right)=$ $\operatorname{genus}(\partial M)>0$, so $\partial M^{\prime}$ is compressible in $N$. Such a compressing disk $E$ cannot lie inside $M^{\prime}$, by construction, so it lies in the connected manifold $N-M^{\prime}$; let $M_{1}$ be the component of $M^{\prime}$ on whose boundary $\partial E$ lies. Since each disk in $\mathbf{D}$ was separating, $M$ has the simple topological description that it is the boundary-connect sum of the components of $M^{\prime}$. So $M$ can easily be reconstructed from $M^{\prime}$ in $N-M^{\prime}$ by doing boundary connect sum along arcs connecting each component of $M^{\prime}-M_{1}$ to $M_{1}$ in $N-\left(M^{\prime} \cup E\right)$. After this reimbedding of $M, E$ is a compressing disk for $\partial M$ that lies outside $M$, so we can conclude the proof via one of the previous cases.

\section{Aligned Fox Reimbedding}

Now we combine results from the previous two sections and consider this question: If $M$ is a connected 3 -submanifold of a non-Haken manifold $N$ and $M$ is $\partial$-reducible, to what extent can a reimbedding of $M$, so that its complement is standard, have its $\partial$-reducing disks aligned with meridian disks of its complement. Obviously non-separating disks in $M$ cannot have boundaries matched with meridian disks of $N-M$, since $N$ contains no non-separating surfaces. But at least in the case when $\partial M$ is connected, this is the only restriction.

Definition 8. For $M$ a compact irreducible orientable 3-manifold, define a disjoint collection of separating $\partial$-reducing disks $\mathbf{D} \subset M$ to be full if each component of $M-\mathbf{D}$ is either a solid torus or is $\partial$-irreducible.

For $M$ reducible, $\mathbf{D} \subset M$ is full if there is a prime decomposition of $M$ so that for each summand $M^{\prime}$ of $M$ containing the boundary, $\mathbf{D} \cap M^{\prime}$ is full in $M^{\prime}$.

$M \subset N$ a 3 -submanifold is aligned to a standard complement if the complement of $M$ is standard and there is a (complete) collection of reducing spheres $\mathbf{S}$ for $\partial M$ so that $\mathbf{S} \cap M$ is a full collection of $\partial$-reducing disks for $M$. 
There is a uniqueness theorem, presumably well known, for full collections of disks, which is most easily expressed for irreducible manifolds:

Lemma 9. Suppose $M$ is an irreducible orientable 3-manifold with boundary and $M$ is expressed as a boundary connect sum in two ways: $M=M_{1} \natural M_{2} \curvearrowleft \ldots \emptyset M_{n}=$ $M_{1}^{*} \curvearrowleft M_{2}^{*} \downarrow \ldots \curvearrowleft M_{n^{*}}^{*}$, where each $M_{i}, M_{j}^{*}$ is either a solid torus or $\partial$-irreducible. Then, after rearrangement, $n^{*}=n$ and $M_{i} \cong M_{i}^{*}$.

Proof. One can easily prove the theorem from first principles, along the lines of, e.g. [H. Theorem 3.21], the standard proof of the corresponding theorem for a connected sum. But a cheap start is to just double $M$ along its boundary to get a manifold $D M$. The decompositions above double to give connected sum decompositions of $D M$ in which each factor consists of either $S^{1} \times S^{2}$ or the double of an irreducible, $\partial$-irreducible manifold, which is then necessarily irreducible. Then $\underline{\mathrm{H}}$, Theorem 3.21] implies that $n=n^{*}$ and that the two original decompositions of $M$ also each contain the same number of solid tori. After removing these, we are reduced to the case in which the only $\partial$-reducing disks in $M$ are separating and $n^{*}=n$.

Following the outline suggested by the proof of [H] Theorem 3.21], choose a disk $D$ that separates $M$ into the component $M_{n}$ and the component $M_{1} \sharp M_{2} \curvearrowleft \ldots \boxminus M_{n-1}$. Choose disks $E_{1}, \ldots, E_{n-1}$ that separate $M$ into the components $M_{1}^{*}, M_{2}^{*}, \ldots M_{n^{*}}^{*}$. Choose the disks to minimize the number of intersection components in $D \cap\left(\bigcup\left\{E_{i}\right\}\right)$. Since each manifold is irreducible and $\partial$-irreducible, a standard innermost disk, outermost arc argument (in $D$ ) shows that in fact $D$ is then disjoint from $\left\{E_{i}\right\}$, so $D \subset M_{n}^{*}$ (say). Since $M_{n}^{*}$ is $\partial$-irreducible, $D$ is $\partial$-parallel in $M_{n}^{*}$. So in fact (with no loss of generality) $M_{n} \cong M_{n}^{*}$ and $M_{1} \curvearrowleft M_{2} \downarrow \ldots \natural M_{n-1} \cong M_{1}^{*} \curvearrowleft M_{2}^{*} \downarrow \ldots \natural M_{n-1}^{*}$. The result follows by induction.

Theorem 10. Let $N$ be a closed orientable irreducible non-Haken 3-manifold, and $M$ be a connected compact 3-submanifold of $N$ with connected boundary. Then $M$ can be reimbedded in $N$ with standard complement so that $M$ is aligned to the standard complement.

Proof. The proof is by induction on the genus of $\partial M$. Unless $M$ has a separating $\partial$-reducing disk, there is nothing beyond the result of Theorem 7 to prove. So we assume that $M$ does have a separating $\partial$-reducing disk; in particular, the genus of $\partial M$ is $g \geq 2$. We inductively assume that the theorem has been proven whenever the genus of $\partial M$ is less than $g$.

The first observation is that it suffices to find an embedding of $M$ in $N$ so that there is some reducing sphere $S$ for $\partial M$ in $N$, for such a reducing sphere divides $J=N-M$ into two components $J_{1}$ and $J_{2}$. Apply the inductive hypothesis to $M \cup J_{1}$ to reimbed it with an aligned complement $J_{2}^{\prime}$. Notice that by a standard innermost disk argument, the reducing spheres can be taken to be disjoint from $S$. After this reimbedding, apply the inductive hypothesis to $M \cup J_{2}^{\prime}$ to reimbed it so that its complement $J_{1}^{\prime}$ is aligned. After this reimbedding, $M$ has aligned complement $J_{1}^{\prime} \cup_{S-M} J_{2}^{\prime}$.

Our goal then is to find a reimbedding of $M$ so that afterwards $\partial M$ has a reducing sphere. First use Theorem 7 to reimbed $M$ in $N$ so that its complement $J$ is standard, i.e. either a handlebody or $N \#$ (handlebody). Since $M$ is $\partial$-reducible, Lemma 3 applies: either $M$ is itself a handlebody (in which case the required reimbedding of $M$ is easy) or there are disjoint compressing disks $D$ in $J$ and $E$ in $M$. Since $J$ is standard, $D$ can be chosen to be non-separating in $J$. Then $\partial E$ is 
not homologous to $\partial D$ in $\partial M$, so $\partial E$ is either separating in $\partial M$ or non-separating in $\partial M-\partial D$. In the latter case, two copies of $E$ can be banded together along an arc in $\partial M-\partial D$ to create a separating essential disk in $M$ that is disjoint from $D$. The upshot is that we may as well assume that $D \subset J$ is non-separating and $E \subset M$ is separating.

Add a 2-handle to $M$ along $D$ to get $M^{\prime}$, still with standard complement $J^{\prime}$. Dually, $M$ can be viewed as the complement of the neighborhood of an $\operatorname{arc} \alpha \subset M^{\prime}$. If $\partial E$ is inessential in $\partial M^{\prime}$, it bounds a disk $D^{\prime}$ in $J^{\prime} \subset J$. Then the sphere $D^{\prime} \cup E$ is a reducing sphere for $M$ as required. So we may as well assume that $\partial E$ is essential in $\partial M^{\prime}$ and of course still separates $M^{\prime}$. By the inductive assumption, $M^{\prime}$ can be embedded in $N$ so that its complement is aligned, but note that this does not immediately mean that $\partial E$ itself bounds a disk in $N-M^{\prime}$. Let $\mathbf{S}$ be a complete collection of reducing spheres for $\partial M^{\prime}$ intersecting $M^{\prime}$ in a full collection of disks.

$E$ divides $M^{\prime}$ into two components, $U$ and $V$ with, say, $\alpha \subset U$. If $M^{\prime}$ is reducible (i.e. contains a punctured copy of $N$ ) an innermost (in $E$ ) disk argument ensures that the reducing sphere is disjoint from $E$. By possibly tubing $E$ to that reducing sphere, we can ensure that the $N$-summand, if it lies in $M^{\prime}$, lies in $U \subset M^{\prime}$. That is, we can arrange that $V$ is irreducible. $E$ extends to a full collection of disks in $M^{\prime}$, with the new disks dividing $U$ and $V$ into $\partial$-connected sums: $U=U_{1} \curvearrowleft \ldots \measuredangle U_{m}, V=$ $V_{1} \natural \ldots \measuredangle V_{n}, m, n \geq 1$, with each $U_{i}, V_{j}$ either $\partial$-irreducible or a solid torus (with one of the $U_{i}$ possibly containing $N$ as a connect summand). By Lemma 9, some component $V^{\prime}$ of $M^{\prime}-\mathbf{S}$ is homeomorphic to $V_{n}$. Tube together all components of $\mathbf{S}$ incident to $V^{\prime}$ along arcs in $\partial V^{\prime}$ to get a reducing sphere $S^{\prime}$ dividing $M^{\prime}$ into two components, one homeomorphic to $V_{n}$ and the other homeomorphic to $U \curvearrowleft V_{1} \downarrow V_{2} \natural \ldots \natural V_{n-1}$. The latter homeomorphism carries $\alpha \subset U$ to an arc $\alpha^{\prime}$ that is disjoint from the reducing sphere $S^{\prime}$. Then $M^{\prime}-\eta\left(\alpha^{\prime}\right)$ is homeomorphic to $M$ and admits the reducing sphere $S^{\prime}$. In other words, the reimbedding of $M$ that replaces $M^{\prime}-\eta(\alpha)$ with $M^{\prime}-\eta\left(\alpha^{\prime}\right)$ makes $\partial M$ reducible in $N$, completing the argument.

Corollary 11. Given $M \subset N$ as in Theorem 10, suppose $\mathbf{D}$ is a full set of disks in $M$. Then, with at most one exception, each component of $M-\mathbf{D}$ embeds in $S^{3}$.

Proof. Following Theorem 10 reimbed $M$ in $N$ with the standard complement so that $M$ is aligned to the standard complement. Then there is a collection $\mathbf{S}$ of disjoint spheres in $N$ so that, via Lemma $9-\mathbf{S}$ and $M-\mathbf{D}$ are homeomorphic. Since $N$ is irreducible, each component but at most one of $N-\mathbf{S}$ is a punctured 3 -ball. Finally, each component of $N-\mathbf{S}$ contains at most one component of $M-\mathbf{S}$ since each component of $\mathbf{S}$ is separating.

\section{REFERENCES}

[CG] Casson, A. J.; Gordon, C. McA., Reducing Heegaard splittings. Topology Appl. 27 (1987), no. 3, 275-283. MR0918537|(89c:57020)

[F] Fox, Ralph H. On the imbedding of polyhedra in 3-space. Ann. of Math. (2) 49, (1948). 462-470. MF 0026326 (10:138c)

[H] Hempel, John, 3-Manifolds, Ann. Math. Studies 86 (Princeton University Press, Princeton, NJ, 1976). MR0415619 (54:3702)

[MT] Menasco, W.; Thompson, A. Compressing handlebodies with holes. Topology 28 (1989), no. 4, 485-494. MR1030989 (91b:57010) 
[ST] Scharlemann, Martin; Thompson, Abigail, Thin position for 3-manifolds. Geometric topology (Haifa, 1992), 231-238, Contemp. Math., 164, Amer. Math. Soc., Providence, RI, 1994. MR 1282766 (95e:57032)

[W] Waldhausen, Friedhelm, Heegaard-Zerlegungen der 3-Sphäre. (German) Topology 7 1968, 195-203. MF 0227992(37:3576)

Department of Mathematics, University of California, Santa Barbara, California 93106

E-mail address: mgscharl@math.ucsb.edu

Department of Mathematics, University of California, Davis, California 95616

E-mail address: thompson@math.ucdavis.edu 